\title{
Vitis OneGenE: A Causality-Based Approach to Generate Gene Networks in Vitis vinifera Sheds Light on the Laccase and Dirigent Gene Families
}

\author{
Stefania Pilati ${ }^{1, *} \mathbb{0}$, Giulia Malacarne ${ }^{1} \oplus$, David Navarro-Payá ${ }^{2}{ }^{\mathbb{D}}$, Gabriele Tomè ${ }^{3}$, Laura Riscica ${ }^{4}$,

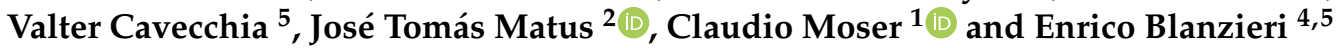 \\ 1 Research and Innovation Centre, Department of Genomics and Biology of Fruit Crops, Fondazione Edmund \\ Mach, 38098 San Michele all'Adige, Italy; giulia.malacarne@fmach.it (G.M.); claudio.moser@fmach.it (C.M.) \\ 2 Institute for Integrative Systems Biology (I2SysBio), Universitat de València-CSIC, \\ 46908 Paterna, Valencia, Spain; david.navarro.paya@gmail.com (D.N.-P.); tomas.matus@uv.es (J.T.M.) \\ 3 Centre for Integrative Biology (CIBIO), University of Trento, 38123 Trento, Italy; \\ gabriele.tome@studenti.unitn.it \\ 4 Department of Information Engineering and Computer Science, University of Trento, 38123 Trento, Italy; \\ laura.riscica@studenti.unitn.it (L.R.); enrico.blanzieri@unitn.it (E.B.) \\ 5 CNR-Institute of Materials for Electronics and Magnetism, 38123 Trento, Italy; valter.cavecchia@imem.cnr.it \\ * Correspondence: stefania.pilati@fmach.it; Tel.: +39-0461-615405
}

\section{check for} updates

Citation: Pilati, S.; Malacarne, G.; Navarro-Payá, D.; Tomè, G.; Riscica,

L.; Cavecchia, V.; Matus, J.T.; Moser,

C.; Blanzieri, E. Vitis OneGenE: A

Causality-Based Approach to

Generate Gene Networks in Vitis vinifera Sheds Light on the Laccase and Dirigent Gene Families.

Biomolecules 2021, 11, 1744. https:// doi.org/10.3390/biom11121744

Academic Editor: Attila Fehér

Received: 9 October 2021

Accepted: 16 November 2021

Published: 23 November 2021

Publisher's Note: MDPI stays neutral with regard to jurisdictional claims in published maps and institutional affiliations.

Copyright: (c) 2021 by the authors. Licensee MDPI, Basel, Switzerland. This article is an open access article distributed under the terms and conditions of the Creative Commons Attribution (CC BY) license (https:// creativecommons.org/licenses/by/ $4.0 /)$.

\begin{abstract}
The abundance of transcriptomic data and the development of causal inference methods have paved the way for gene network analyses in grapevine. Vitis OneGenE is a transcriptomic data mining tool that finds direct correlations between genes, thus producing association networks. As a proof of concept, the stilbene synthase gene regulatory network obtained with OneGenE has been compared with published co-expression analysis and experimental data, including cistrome data for MYB stilbenoid regulators. As a case study, the two secondary metabolism pathways of stilbenoids and lignin synthesis were explored. Several isoforms of laccase, peroxidase, and dirigent protein genes, putatively involved in the final oxidative oligomerization steps, were identified as specifically belonging to either one of these pathways. Manual curation of the predicted sequences exploiting the last available genome assembly, and the integration of phylogenetic and OneGenE analyses, identified a group of laccases exclusively present in grapevine and related to stilbenoids. Here we show how network analysis by OneGenE can accelerate knowledge discovery by suggesting new candidates for functional characterization and application in breeding programs.
\end{abstract}

Keywords: OneGenE; stilbenoids; lignin; causal inference; gene network; transcriptomics; peroxidase; laccase; dirigent protein

\section{Introduction}

The availability of an ever-increasing number of Vitis ssp. transcriptomic data and the creation and maintenance of integrated and comprehensive compendia [1,2] are paving the way to developing system biology approaches in the grapevine. Reusing omics data, in line with the GO FAIR guiding principles [3], represents an advantageous opportunity to drive knowledge discovery from existing data. In particular, gene network reconstruction is receiving growing attention. Gene regulatory networks (GRNs) provide a convenient way of representing as graphs the functional interactions (as edges) of the genes (as nodes) of an organism [4]. Besides highlighting gene correlations, this representation can contribute to hub gene identification, gene function prediction, data integration from different omics techniques, and comparative gene network analyses across species [5]. One approach to finding correlations among genes is based on the "guilt by association" assumption that genes with similar expression profiles are likely to be functionally related. This approach, primarily based on Pearson's correlation and mutual information analyses, produces 
weighted gene co-expression networks (WGCNs). In grapevine, WGCN analysis has been applied in many cases, to analyze a single or a few experiments [6-12]. Recently, an improved version based on the aggregation of multiple WGCN analyses performed on different datasets (AggWGCN) has been proposed to identify gene networks using a selection of public transcriptomic data [13,14]. For co-expression analyses, the sample size and quality-the number and the kind of experiments selected to build the dataset-are important features to consider for the algorithm performance.

A different approach is represented by causal discovery analyzing observational data or data collected on various conditions. The search through causal relationships among co-related variables (here transcript expression levels) aims to go beyond simple correlation, simplifying the correlation graph by deleting relationships that appear to be independent once a separation set of variables is applied. A notable example of this approach is the PC algorithm [15], whose application to transcriptomic data could be done by iteratively subsetting the variables and combining the results afterward. This approach is adopted by NES $^{2} R A$; a causal inference method applied to the entire VESPUCCI database to expand known local gene networks (LGNs) [16]. The high number of PC-algorithm runs required by the iterative subsetting is computed in a distributed way by the gene@home project that exploits the BOINC platform for volunteer computation [17]. NES ${ }^{2}$ RA produces an association network whose edges represent putative direct interactions between genes, as an interaction still exists despite a high number of attempts to remove it by individuating a separation set of variables.

Despite NES ${ }^{2}$ RA's reported ability to identify gene interactions starting from a nondedicated large transcriptomic dataset, some limitations related to its practical use were, however, evident. First, the heavy computation of the LGN expansion cannot be requested directly by the user and may require up to several days to complete, even with the use of the BOINC distributed computing platform. Second, the analyses are not reusable, as they are very likely to be unique elaborations, given that the number of possible gene combinations is exponential in the size of a genome. Finally, a priori knowledge of the LGN is not always the starting point of an inquiry; researchers often have good candidate genes and need to first refine the LGN before expanding it. To overcome these difficulties, in the present work, we adopted the OneGenE approach, which aims to expand each single gene in an organism. OneGenE systematically runs-single gene NES ${ }^{2} \mathrm{RA}$ expansions with fixed parameters and combines them afterward to simulate LGN expansions. In this way, the single-gene expansions can be used multiple times, thus effectively reducing the computational effort [18].

Stilbenoids are a group of polyphenols acting as phytoalexins in grapevine and other species due to their antifungal properties [19]. They derive from the STS-catalyzed synthesis of trans-resveratrol, which can be further modified by enzyme-mediated methylation, hydroxylation, glycosylation, or oligomerization [20]. Stibenoid synthesis often occurs upon biotic stresses, and oligomers show higher toxicity than monomers [20,21]. The STS of the grapevine gene family has been characterized as encompassing 41 predicted isoforms falling into three main phylogenetically distinct groups, specifically group A clustered on chromosome 10 and groups B and C on chromosome 16 [22]. The high copy number of STS genes in the grapevine genome suggests a high dosage requirement of the encoded protein as well as a functional specialization of the different isoforms. It is known that resveratrol accumulation in grapevine is strictly controlled at the transcriptional level by the regulation of STS isoforms expression, specifically activated during development [23] and in response to biotic and abiotic stresses [22,24,25]. The first study aiming to discover the VviSTS regulators has identified two R2R3-MYB factors, VviMYB14 and VviMYB15, explicitly activating the promoters of VviSTS41 and VviSTS29 genes in transient reporter assays [25]. Other regulators have been identified since then, and we refer the reader to the Results section, where they are presented in detail to support the validation of the approach. Neither the enzymes catalyzing resveratrol oxidative polymerization into dimers or higher degree oligomers, nor their transcriptional regulators, have so far been identified 
and characterized. Interestingly, six peroxidase (PRX) genes (VviPrxIII08a, VviPrxIII08b, VviPrxIII15a, VviPrxIII21a, VviPrxIII23a, and VviPrxIII34a) and two laccase (LAC) genes (VIT_218s0001g02400 and VIT_218s0001g02410) were proposed as possibly involved, based on their localization in QTL regions related to monomeric and oligomeric stilbenoid and $\varepsilon$-viniferin accumulation, respectively [26]. Moreover, the involvement of dirigent proteins (DIR) has been suggested due to the stereospecificity of stilbene oligomers, but it has not been proven so far [21,27].

Lignin is a phenylpropanoid-derived polymer found in specific cell types of vascular plants, particularly those with secondary thickened cell walls involved in the transport of water or mechanical support. Lignin is derived from the oxidative bimolecular radical coupling of three monolignols - coniferyl, sinapyl, and p-coumaryl alcohols-in a reaction catalyzed by LAC and class III PRX [28] and assisted by DIR proteins, which ensure the stereoselectivity of the reaction $[29,30]$. The three monolignols are synthesized through a branch of the phenylpropanoid pathway, initiated by the enzymes cinnamoyl CoA reductase (CCR) and hydroxycinnamoyl transferase (HCT). Different members of the PRX III gene family have been functionally characterized as associated with lignification in Arabidopsis [28,31]. Phylogenetic reconstruction of the grapevine PRX gene family, together with the one from Arabidopsis, allowed us to identify the grapevine putative orthologs of AtPrx members involved in lignification [26]. DIR families are widespread in most plant species, from herbaceous to perennials, monocots, and dicots [29,30]. However, it has not been characterized yet in the grapevine. The biosynthesis of lignin is transcriptionally controlled through the regulation of genes encoding enzymes involved in monolignol biosynthesis and lignin polymerization. High-level regulators are members of the NAC and MYB families, as elucidated in Arabidopsis; however, identification of the whole regulatory cascade is still ongoing [32].

In the grapevine, some putative R2R2 MYB TFs involved in lignin biosynthesis have been identified by GCN and phylogenetic analyses based on Arabidopsis comparison [33]. The VviMYB103 (VIT_02s0025g00320) and VviMYB26 (VIT_09s0002g01670) were identified as the putative orthologs of AtMYB103, positively regulating ferulate 5-hydroxylase (F5H) expression and syringyl lignin biosynthesis in the stem [34], and AtMYB26 involved in the formation of secondary thickening in the anther endothecium [35]. VviMYB190 (VIT_00s1241g00010) and VviMYB191 (VIT_16s0022g01210) clustered with AtMYB85, associated with the regulation of lignin biosynthesis [36]. Finally, VviMYB185 (VIT_06s0004g02110) and VviMYB186 (VIT_13s0064g00960) belong to the same subgroup of AtMYB46, associated with the regulation of cell wall biosynthesis [37]. The six MYBs putatively related to lignin synthesis have been used as input genes for OneGenE to identify LAC, PRX, and DIR isoforms possibly involved in lignin synthesis.

In the present work, first, we offer a proof of concept for the OneGeneE approach by reconstructing the well-characterized GRN of grapevine STS and comparing our results with the state-of-the-art available knowledge. We also present the OneGenE website, where the expansion lists for any grapevine gene are made available and describe the procedure required to generate association networks starting from a set of genes. Second, we present the application of the OneGenE method to two uncharacterized grapevine pathways-the stilbenoids and lignin biosynthesis - to show the potential of the OneGeneE approach. Finally, the results obtained with OneGenE are integrated with the phylogenetic analysis of the Vitis LAC and DIR families shedding light on group functional specialization within these families.

\section{Materials and Methods}

\subsection{Computation of OneGenE Expansion Lists}

The expansion of each gene of the Vitis vinifera genome originated from the run of the algorithms of the OneGenE system [18,38]. Each expansion procedure consisted of 2000 iterations of our C++ implementation (https:/ / bitbucket.org/francesco-asnicar/pc-boinc/, last accessed on 16 June 2020) of the PC-algorithm skeleton procedure [39] $(\alpha=0.05)$ 
to 29 sets of 1000 variables (genes), which included the gene to be expanded, and a random subset of 999 genes sampled without replacement. The blocks scheme of the OneGenE architecture is shown in Blanzieri et al. 2020, Figure 1 [18]. The input data were extracted from the $28,013 \times 1131$ normalized expression data matrix initially obtained from the VESPUCCI repository [1], filtered, and preprocessed [16]. Each expansion list is ordered with respect to the relative frequency, namely $\mathrm{F}_{-}$rel = \#times the gene is present in the output of the PC-algorithm/\# times the gene is present in the input of the PC-algorithm. Overall, the computation of the expansion lists of all the genes required $28,013 \times 29 \times 2000=1,624,754,000$ runs of the PC algorithm, each run taking $5.63 \mathrm{~s}$ on average on our reference machine (Intel i7-4770K, Ubuntu). Therefore, the computation was done within the gene@home project on the volunteer distributed computation platform TN-Grid (20 TeraFLOPS on average) [17], powered by BOINC [40] software.

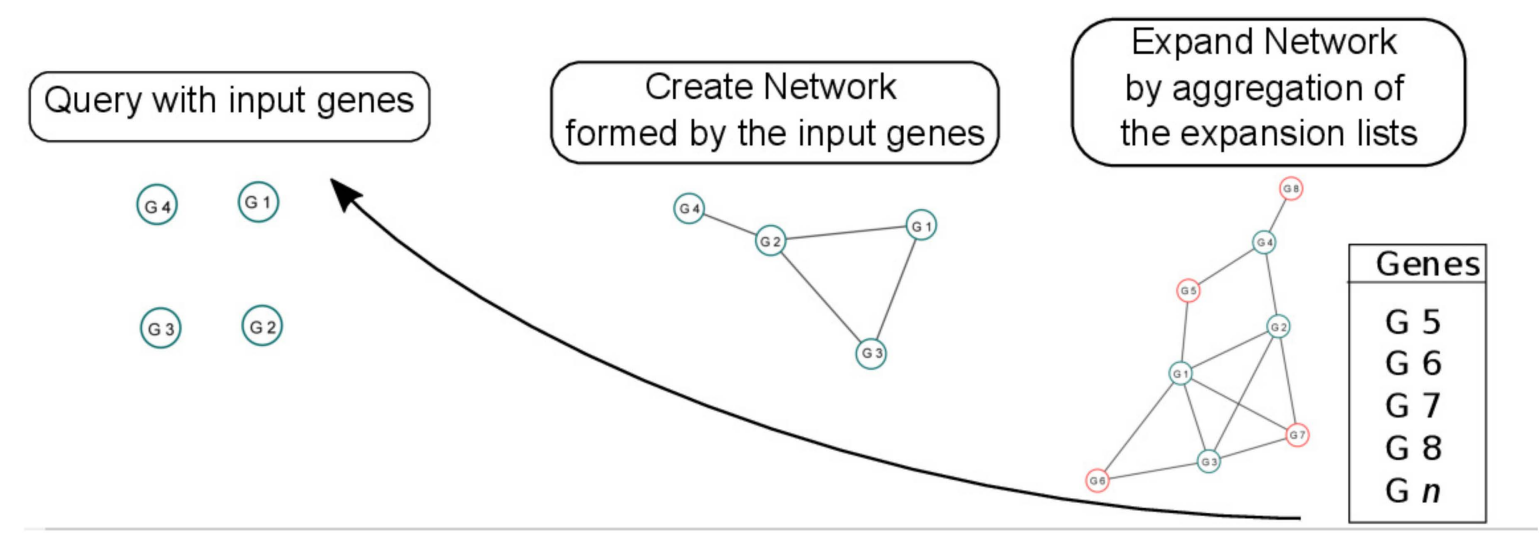

Figure 1. A general pipeline of the analysis to reconstruct a gene network.

The OneGenE expansion lists were annotated using the catalogue.INTEGRAPEv2 (http:/ / www.integrape.eu/index.php/resources/genes-genomes, 19 October 2021), published works of genes families already characterized in grapevine, Vitisnet gene annotation [41], and the VCost.v3 annotation [42]. The "gene clusters", as defined in the VESPUCCI database [1], could not be automatically annotated.

\subsection{Programs Developed to Reconstruct Gene Networks Using OneGenE Output}

Three programs have been developed in Python v3.8.10 (https://www.python.org/) to create, expand, visualize, and analyze gene networks starting from the OneGenE expansion lists. The first two tools have been implemented on the website (http://vitis. onegenexp.eu).

\subsubsection{Create Network}

This program computes the interactions within a set of input genes by analyzing their OneGenE expansion lists. As input, it takes a compressed file (.zip) containing the OneGenE expansion lists, downloaded from the OneGenE website (vitis.onegenexp.eu). The user must set a minimum threshold of relative frequency, which defines the number of genes considered for the analysis. The analysis is performed in pairs: for each pair of input genes, the mutual presence of each gene in the trimmed expansion list of the other one is verified, and the mean of their relative frequency values is computed. The tool produces two tables, one for the edges/interactions and one for the nodes/genes, an image file with a static representation of the gene network as an undirected weighted graph, and a .json file which can be imported into Cytoscape 3.8.2 [43] or Cytoscape.js [44] for improved visualization of the gene network. 


\subsubsection{Expand Network}

This program aggregates the OneGenE expansion lists of a set of input genes according to the criteria available to the user. In addition to the compressed file with the expansion lists, it requires a text file input with the V1 IDs of the genes to be aggregated. The aggregation can be performed according to the following four criteria: (i) by considering the genes above a threshold of relative frequency (-frel -f) or (ii) of rank value (-rank); (iii) by considering the genes belonging to a functional category according to the Vitisnet annotation (-pattern -f), for example, setting "-pattern vv60" will aggregate the genes annotated as transcription factors present in the expansion lists; (iv) by considering the genes shared among the input genes (-shared -f). The output will be composed of the same types of files explained for the first tool ("Create network").

\subsubsection{Network Analysis}

This program performs Gene Ontology (GO) and promoter motifs enrichment analyses. By choosing the "-topGO" option, the program performs an enrichment analysis based on the Biological Process and Molecular Function categories of the GO, using the TopGO Bioconductor package [45]. It requires a text file with the V1 ids of the genes to be analyzed as input and a GO annotation file of the whole $V$. vinifera genome. The output consists of a table and a graphical representation of the enriched categories, ranked according to their statistical significance. By choosing the "-DREME" option, the program will prepare a file containing the $1-\mathrm{kb}$ promoters of the genes in FASTA format. This file is suitable for promoter motifs enrichment analysis using the STREME tool of MEME suite [46]. The program requires the same text files input with the V1 IDs of the genes to be analyzed and a file containing the $1-\mathrm{kb}$ promoters of the whole grapevine genome (12X.v1).

\subsection{Genome-Wide Search and Gene Model Curation of LAC and DIR Gene Families}

To retrieve the complete list of isoforms for the $L A C$ and DIR gene families, HMMER search based on Pfam domains was performed on the 12X.v2 genome assembly (VCost.v3 annotation) [42]. Three different multicopper oxidase Pfam domains (PF00394, PF007731, PF07732) and the dirigent Pfam domain (PF03018) were used for LAC and DIR identification, respectively. The genes containing at least one Pfam domain with an e-value $\leq 10^{-5}$ were treated as potential $L A C$ or DIR isoforms. The Web Apollo genome editing platform [47] was used to manually curate their gene models based on public RNA-seq data, as a correct coding sequence was a prerequisite for a correct phylogenetic analysis.

\subsection{Phylogenetic Analysis of the LAC and DIR Gene Families}

The protein sequences of the manually curated genes were then subjected to phylogenetic analysis, together with Arabidopsis genes. For the LAC analysis, the 17 LACs present in the Arabidopsis genome were included as well as the selected members of other multicopper oxidase families sharing similar copper-centered protein domains, such as Ascorbate Oxidases (AOs) and SKU5 Similar (SKS) proteins. Nitrite reductases (NIRs) are multicopper oxidases, but an initial inspection involving multiple protein alignments showed that none of the potential $V$. vinifera $L A C$ genes appeared to correspond to this multicopper oxidase family. Hence, members of this family from Arabidopsis were not included in the final phylogenetic analysis. Low Phosphate Root (LPR) multicopper oxidases were used as an outgroup. The complete list of Arabidopsis and Vitis genes used for the phylogenetic analysis is available in Table S6. The molecular evolution model selected for LAC analysis was 'WAG $+\mathrm{F}+\mathrm{G}$ ' for the bayesian phylogenetic inference carried out with MrBayes [48]. A total of three million generations were run, reaching an average standard deviation of split frequencies of 0.010 and full chain convergence. For the DIR family phylogenetic analysis, all 25 Arabidopsis DIR proteins were included (Table S7), and the model selected was 'JTT $+G^{\prime}$ '. A total of one million generations were run, reaching an average standard deviation of split frequencies of 0.0075 and full chain convergence. 


\subsection{Data and Code Availability}

OneGenE annotated lists can be downloaded as compressed files from the website vitis.onegenexp.eu. The reconstructed networks computed on the website can be downloaded as well, both as images and in a Cytoscape-compliant format. The programs developed in python to reconstruct and analyze the gene networks are available at https: / / github.com/gabrieletome/vitis_tools (last updated on 1 November 2021). In addition to the code, the repository contains the files used for the analyses presented in the present article as examples.

\section{Results}

\subsection{The Grapevine OneGenE Website}

In order to make OneGenE results available to the community, we built a website, vitis.onegenexp.eu (last update on 19 November 2021). From the "OneGeneE analysis" page, the user can perform a query with a list of genes of interest, using the gene IDs of the 12X.v1 release of the grapevine genome annotation, and download the corresponding expansion lists from the result page. The "Network analysis" link redirects the user to a new page where they have options to either build the network formed by the selected genes ("Create network") or to expand the obtained list to find new interacting genes ("Expand network"). The networks are visualized in the bottom part of the page, with different layouts and the choice of using different IDs for the nodes (12X.v1, VCost.v3, gene symbol). These networks can be exported as images (.png) or as files (.json) for further customization, for example, in Cytoscape [43]. A "Tutorial" page has been created with the STS network analysis described in the present paper as an example.

\subsection{The Stilbene Synthase Gene Regulatory Network as a Proof of Concept}

The reconstruction of the STS GRN represented a suitable case for validating the OneGenE approach because of its prevalent transcriptional regulation and the availability of comparative published studies. The strategy we applied is summarized in Figure 1 and represents a general model also used for the stilbenoid and lignin networks. The process starts with a query on the OneGenE website to retrieve the expansion lists of the input genes, using them to build their network. This network is then expanded by consolidating all the lists into one aggregated list. Although, in principle, this new network can be visualized, too many nodes make the representation less clear. Alternatively, the user can inspect this list and choose the newly identified genes of interest, such as specific transcription factors (TFs) or gene families. This restricted set of genes can be used to query the website and build a network, as previously described. Gene networks are evaluated through enrichment analysis of GO functional categories to provide a general idea of the gene-sets obtained with the expansion analysis.

A query with the 41 VviSTS 12X.v1 IDs allowed the retrieval of 22 expansion lists from the OneGenE website (Table S1), as two isoforms were not present in the VESPUCCI processed dataset, and 21 isoforms were compressed into 4 clusters to overcome the problem of probe mapping ambiguity [1]. These lists were used to create the network formed by VviSTSs, setting a minimum relative frequency threshold of 0.5 to focus on the most direct connections (Figure S1). An almost fully connected network was obtained. Remarkably, the three VviSTS groups identified as A, B, and C in the phylogenetic analysis [22] did not show any mirroring modularity at this level of analysis. As a second step, the STS network was expanded to find its related genes (relative frequency threshold set at 0.5 as above). An aggregated list of 955 genes has been obtained (Table S2), enriched in defense response, biosynthetic process, lignin catabolic process, and oxidation-reduction process, according to the GO biological process categories (Table S3). To produce the STS GRN, the list was manually inspected to identify the TFs. A total of 72 TFs were found, 39 of which connected to at least two VviSTS. This STS GRN has been compared with available published data to validate OneGenE analysis results (Table 1). 
Table 1. List of the transcription factors of the STS GRN identified with OneGenE and mentioned in previous works.

\begin{tabular}{|c|c|c|c|c|c|c|c|}
\hline $\begin{array}{c}\text { N. of Connected } \\
\text { VviSTS }\end{array}$ & 12X.v1 & Gene Name & Reference & $\begin{array}{c}\text { N. of Connected } \\
\text { VviSTS }\end{array}$ & 12X.v1 & Gene Name & Reference \\
\hline 15 & Vitvi11g00165 & VvibHLH045 & {$[49,50]$} & 2 & Vitvi17g00025 & VviERF118 & [49] \\
\hline 14 & Vitvi07g00598 & VviMYB14 & {$[13,49,51]$} & 2 & Vitvi15g00948 & $\begin{array}{l}\text { VvibHLH066, } \\
\text { MYC7E }\end{array}$ & - \\
\hline 13 & Vitvi07g02070 & VviERF098 & {$[49,50]$} & 2 & Vitvi15g00938 & VviMYBPA1 & - \\
\hline 9 & Vitvi14g01523 & VviWRKY43 & {$[13,49,52]$} & 2 & Vitvi08g01412 & VviRAV5 & - \\
\hline 8 & Vitvi08g01931 & VviHsfB3a & {$[49,50]$} & 2 & Vitvi08g01224 & ZFDHHC_8 & [52] \\
\hline 7 & Vitvi05g01732 & VviMYB13 & [49] & 1 & Vitvi14g01617 & C2H2FAM_25 & - \\
\hline 7 & Vitvi17g00309 & VviMYB164 & - & 1 & Vitvi11g00715 & C2H2FAM_28 & - \\
\hline 7 & Vitvi06g00007 & VviNAC44 & {$[49,52]$} & 1 & Vitvi11g00436 & CCCHFAM_12 & - \\
\hline 7 & Vitvi08g00793 & VviWRKY24 & {$[49,50,53]$} & 1 & Vitvi15g00636 & GATA2 & - \\
\hline 7 & Vitvi13g00620 & ZFC3HC4_163 & - & 1 & Vitvi13g01879 & GCN5_18 & - \\
\hline 6 & Vitvi15g01203 & ERF2_1 & [50] & 1 & & IAA22D_2 & - \\
\hline 6 & Vitvi04g02175 & VviJAZ3 & - & 1 & Vitvi06g00167 & JMJD5 & - \\
\hline 6 & Vitvi19g02272 & VviNAC31 & [49] & 1 & Vitvi15g00736 & VviLBDIa2 LBD19 & - \\
\hline 6 & Vitvi12g00076 & VviNAC36 & {$[49,50]$} & 1 & Vitvi13g00085 & VviLBDIc3 LBD21 & {$[50,52]$} \\
\hline 5 & Vitvi05g00523 & GCN5_7 & [50] & 1 & Vitvi00g01060 & VviLBDIc5 & - \\
\hline 5 & Vitvi08g01525 & HMGIY_1 & [52] & 1 & Vitvi01g01921 & MYB3R1_5 & - \\
\hline 5 & Vitvi01g01287 & $\begin{array}{l}\text { VvibHLH001, } \\
\text { BIM3 }\end{array}$ & - & 1 & Vitvi14g00217 & NFYB5_3 & - \\
\hline 5 & Vitvi05g01733 & VviMYB15 & {$[13,49-51]$} & 1 & Vitvi18g01322 & RGL2_1 & - \\
\hline 5 & Vitvi04g00133 & VviWRKY06 & [50] & 1 & Vitvi01g00185 & SAP7_3 & - \\
\hline 5 & Vitvi17g00556 & VviWRKY53 & [49-51] & 1 & Vitvi13g00311 & SCL14_1 & {$[49,52]$} \\
\hline 4 & Vitvi10g00053 & JMJD1B_5 & [50] & 1 & Vitvi07g01762 & TFB3_4 & - \\
\hline 4 & Vitvi19g02273 & VviNAC30 & [52] & 1 & Vitvi07g01529 & VvibHLH038 & - \\
\hline 4 & Vitvi17g00801 & ZFC3HC4_103 & - & 1 & Vitvi15g01027 & VvibZIP40 & - \\
\hline 3 & Vitvi07g02168 & MTERF_10 & - & 1 & Vitvi05g01722 & VviERF109 & - \\
\hline 3 & Vitvi01g00940 & VviWRKY02 & {$[13,49,50]$} & 1 & Vitvi15g01206 & VviERF073 & [49] \\
\hline 3 & Vitvi01g01680 & VviWRKY03 & {$[49,50,52]$} & 1 & Vitvi19g01669 & VviMYB139 & {$[49,52]$} \\
\hline 3 & Vitvi04g00760 & VviWRKY11 & [49] & 1 & Vitvi07g03055 & VviMYB148 & [49] \\
\hline 3 & Vitvi19g00155 & ZFC3HC4_158 & [52] & 1 & Vitvi19g01564 & VviNAC29 & [52] \\
\hline 3 & Vitvi12g00220 & ZFC3HC4_36 & [50] & 1 & Vitvi04g00756 & VviWRKY10 & - \\
\hline 2 & Vitvi10g00572 & - & - & 1 & Vitvi06g01574 & VviWRKY15 & [50] \\
\hline 2 & Vitvi13g01958 & ERUNK_7 & - & 1 & Vitvi10g00063 & VviWRKY29 & {$[49,53]$} \\
\hline 2 & Vitvi09g00064 & VviJAZ4 & - & 1 & Vitvi13g00189 & VviWRKY40 & [52] \\
\hline 2 & Vitvi10g00826 & VviJAZ5 & - & 1 & Vitvi16g01213 & VviWRKY51 & [50] \\
\hline 2 & Vitvi10g01879 & VviJAZ6 & - & 1 & Vitvi05g00368 & ZFC3HC4_164 & - \\
\hline 2 & Vitvi16g00349 & VviERF077 & - & 1 & Vitvi08g00923 & ZFC3HC4_43 & - \\
\hline 2 & Vitvi02g01780 & VviERF076 & [50] & 1 & Vitvi16g02032 & ZFC3HC4_45 & - \\
\hline
\end{tabular}

VviMYB14 and VviMYB15, initially identified by Höll et al. [25], were found as expected among the most connected to VviSTS. VviWRKY24 and 29, previously identified as co-expressed with VviSTS during drought stress [53], were also found by OneGenE, as were the $20 \mathrm{TFs}$ previously identified by GCN analysis and promoter activation assay [49], belonging to the R2R3MYB, WRKY, NAC, bHLH, and ERF families. Recent functional characterization works in a wild grape confirmed the interaction of VqWRKY53 with VqMYB14 and $V q M Y B 15$, affecting VqSTSs activation in V. quinquangularis [51]. A recent transcriptomic analysis-not included in VESPUCCI and thus not used by OneGenE- proving the role of stilbenoid accumulation in plant defense to Plasmopara viticola [50], extensively validated our GRN, as well. Finally, a significant overlap with VviMYB14 and VviMYB15 cistrome analysis was observed [52]. 
Beyond providing suitable proof of concept, the STS GRN here obtained presented several interesting features worth mentioning. The visual representation of the network highlighted a partitioning of VviSTSs around three hubs, namely VviMYB14, the VviWRKY43-53 pair, and the VviMYB13-15-VviWRKY24 triad (Figure 2A). Again, the three groups of VviSTSs appear largely overlapping, with VviMYB14 showing the highest degree of connection to almost all group B STSs. The present network is consistent with the reported activation of VviSTS29 (here in CL_B_2) by VviMYB14 and VviMYB15, alone and in combination with $V v i W R K Y 3$, and by VviWRKY 24 , which have been experimentally demonstrated [25,49]. Moreover, the OneGenE approach identified four members of the Jasmonate ZIM domain (JAZ) TF family, all connected to VviMYB14 (Figure 2B). This is the first time this family of regulators has been reported as associated with the STS GRN. Two genes encoding MYC-type TFs, VvibHLH001-VviBIM3 and VvibHLH066-VviMYC7E (J.T. Matus, personal communication), have been identified too, as inversely related to specific VviSTS isoforms. The former is directly linked to VviJAZ3, while the latter is located in the VviWRKY43-53 subnetwork. Further investigation is required to support their involvement in jasmonate signaling. Finally, two TFs belonging to the Zinc finger C3HC4 type, ZFC3HC4_163 and ZFC3HC4_36, appeared highly connected within the VviWRKY43-53 subnetwork, one of which was also identified by DAP-seq analysis [52].

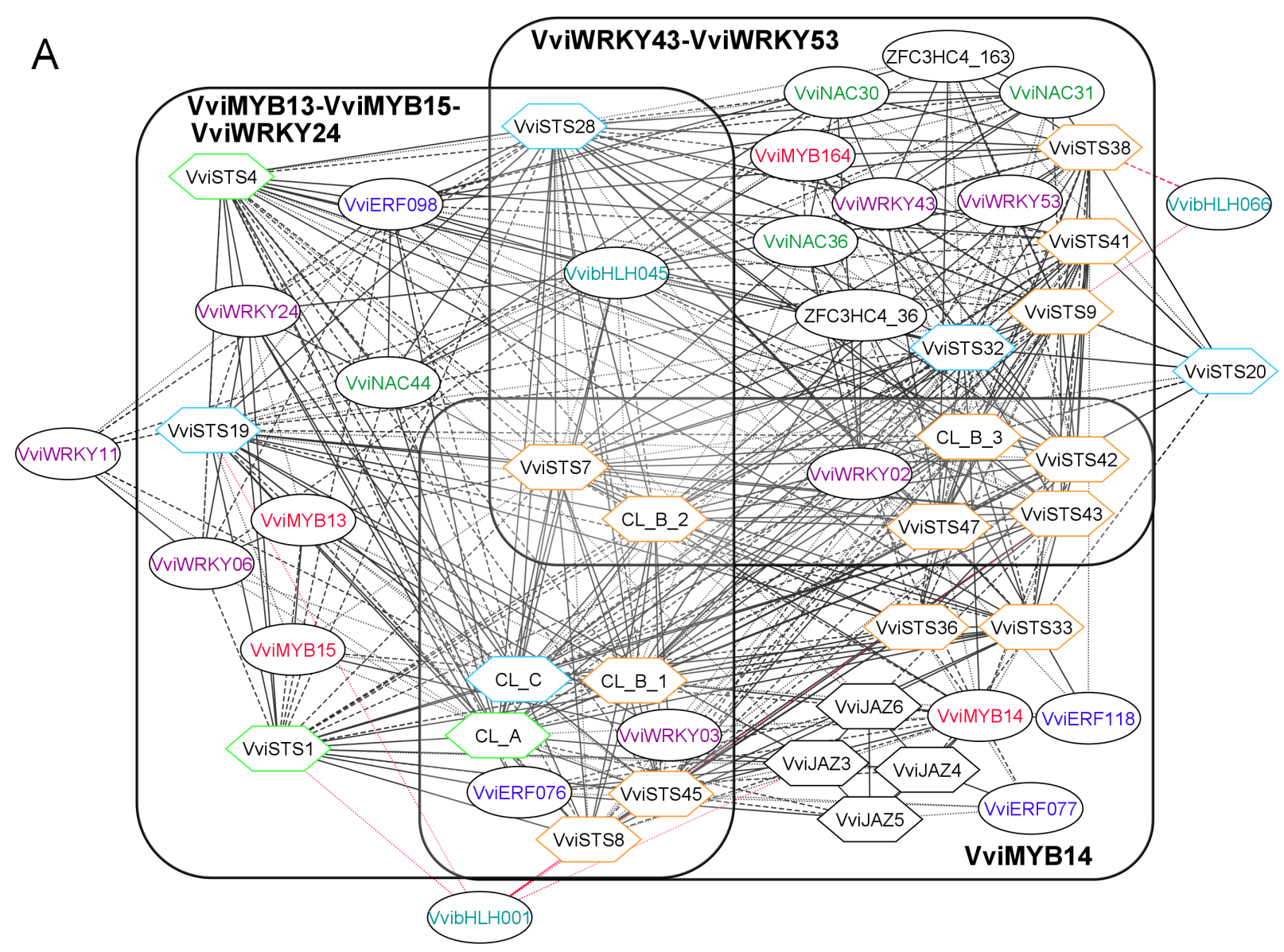

Figure 2. Cont. 


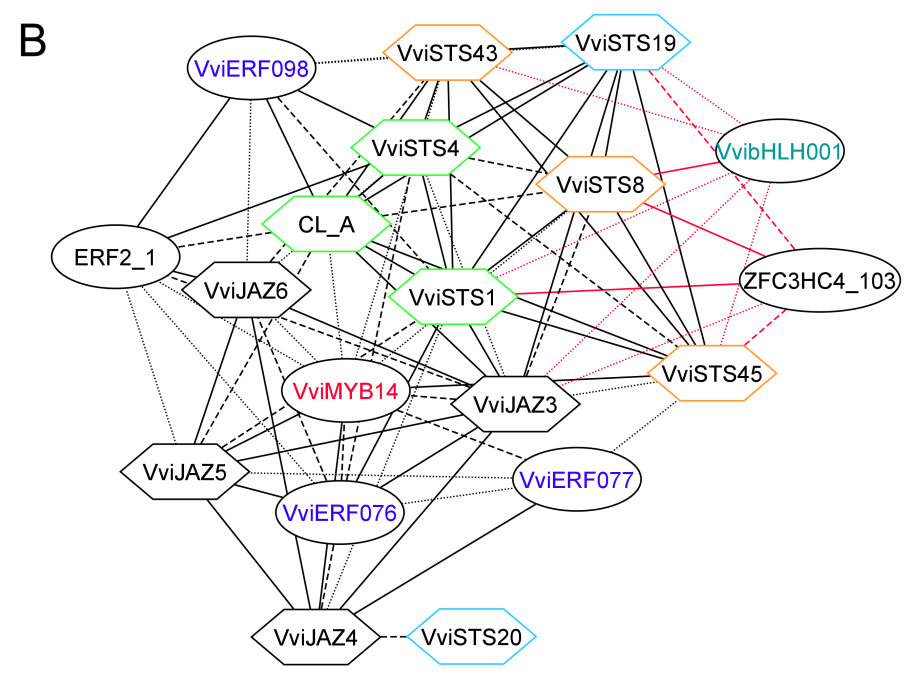

Figure 2. The STS GRN. (A) Partitioning of the STS GRN into three overlapping subnetworks, enclosed in rectangles, centered on VviMYB14, VviWRKY43-53, and VviMYB13-15-VviWRKY24. The STS hexagonal nodes border is colored in green, orange, and light blue according to the phylogenetic groups A, B, and C as defined in [22]. The Edge line type (solid, dashed, or dotted) represents edge weight classes based on the mean relative frequency (decreasing from 1 to 0.5 ). Red edges correspond to negative values of Pearson's correlation coefficient, thus joining anticorrelated genes. (B) The JAZ TFs subnetwork was extracted from the STS GRN. The JAZ TFs adjacent nodes have been extracted from the STS GRN to better visualize this part of the network.

\subsection{Laccase, Peroxidase and Dirigent Proteins Isoforms Involved in the Stilbenoid and Lignin Networks}

As a case study, the OneGenE method was applied to gain more insight into the stilbenoid and lignin pathways. We focused on the gene families putatively involved in the oligomerization of resveratrol and monolignols. Concerning the former, we used the same aggregated list derived from the STS network expansion: it included many genes annotated as LAC, PRX III, and DIR, as well as a glycosyl- and O-methyl-transferases, putatively involved in resveratrol conversion to piceid and pterostilbene (Table S2, Figure 3). For the lignin network reconstruction, we used the six MYB TFs identified in [33] as a query on the OneGenE website to expand them, obtaining an aggregated list of 676 genes (Table S4). They were enriched in the lignin catabolic process and regulation of nitrogen (Table S5), supporting their involvement in lignin metabolism. Several genes annotated as LAC, PRX III, and DIR were found, as expected, in the network (Figure 4).

The first very evident outcome was that both networks included several isoforms of PRX III, LAC, and DIR but altogether distinguished. For PRX class III, phylogenetic analysis and a precise name assignment were already available [26]. This information allowed us to identify six PRX III of class 01-located on chromosome 1-in the stilbenoid network together with two PRXIII of class 22 and one of class 23 . VviSTS20 was connected to all three PRX III classes, the O-methyltransferase and the glycosyltransferase, the latter in a negative way, suggesting that this STS could be involved in pterostilbene accumulation and its oxidative modifications. VviSTS20 also showed a particular regulation, as it was connected only to VviJAZ4 and ZFC3HC4_163 (Figure 2). VviSTS38, 32, 47, and 9 interacted with many class 01 PRX III and DIR and belonged to the VviWRKY43-53 subnetwork. Finally, PRX III and DIR were located distantly in the network, interacting not directly but through STSs nodes, despite being assumed to catalyze consecutive steps of stilbenoids polymerization. Conversely, the lignin network looked more uniformly connected. OneGenE identified two PRX III: one of class 12 and one of class 07. The latter, homolog of AtPRX64, was highly connected to three of the MYB TFs, five LAC, and one DIR gene. 


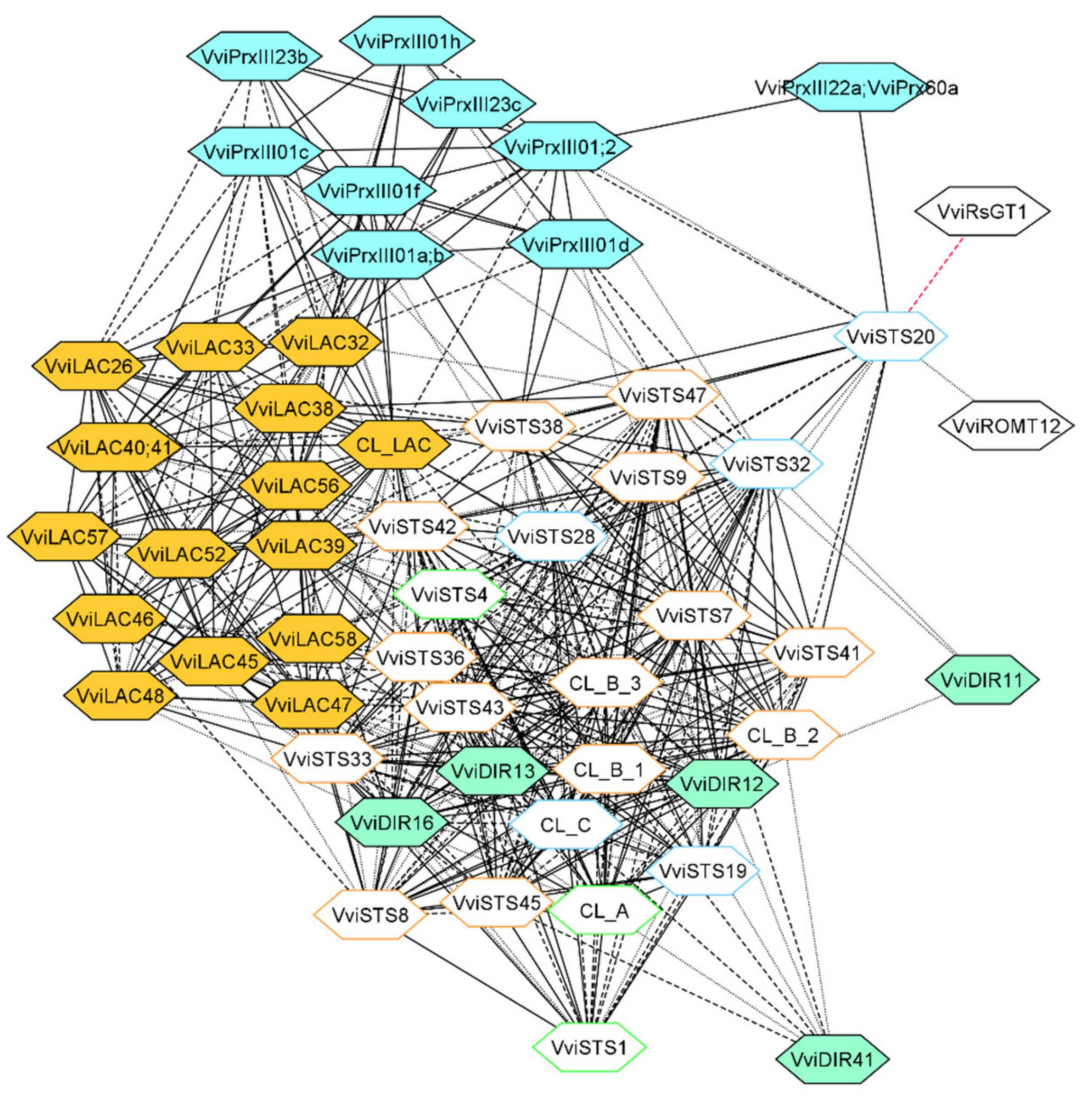

Figure 3. The LAC, PRX, and DIR isoforms of the stilbenoid network. LAC, DIR, and class III PRX nodes are colored in orange, light green, and light blue, respectively. The Edge line type and STS nodes border is defined as in Figure 2.

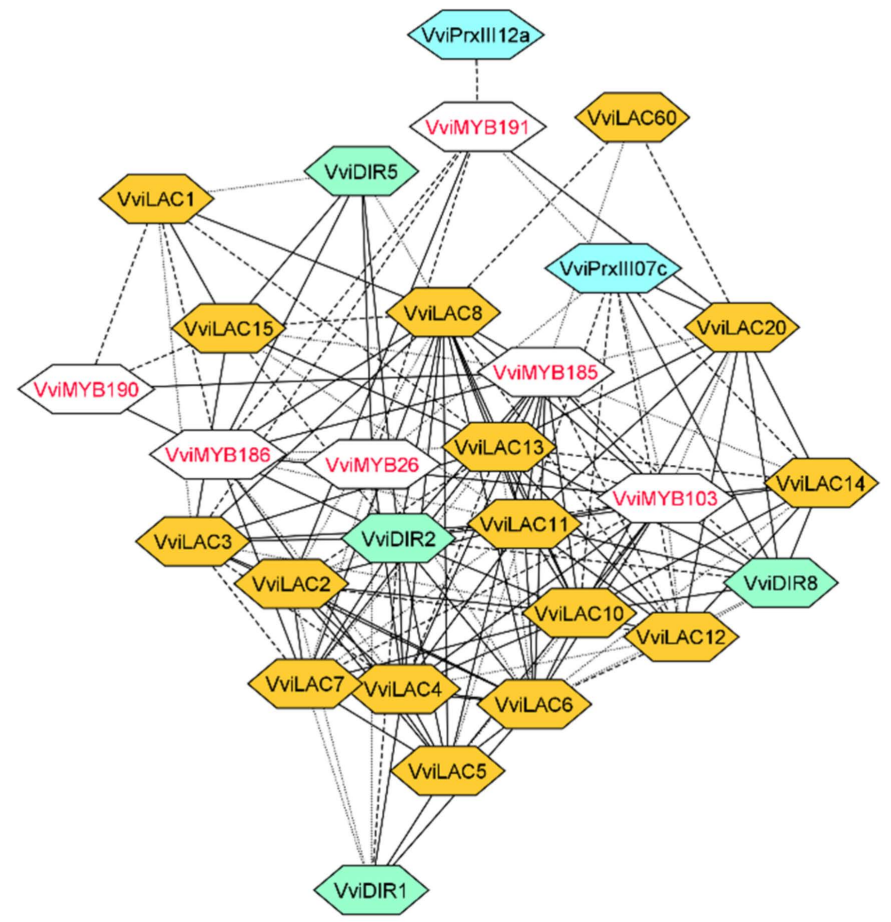

Figure 4. The LAC, PRX, and DIR isoforms of the lignin network. LAC, DIR, and class III PRX nodes are colored in orange, light green, and light blue, respectively. The edge line type and STS nodes border is defined as in Figures 2 and 3. 
A thorough phylogenetic characterization of these families has been undertaken to better describe these networks and provide precise indications on the LAC and DIR isoforms putatively involved in one or the other pathways. A genome-wide search for LAC genes in the 12X.v2 genome assembly identified another 44 multicopper oxidases in addition to the 77 already annotated as LAC in the 12X.v1 (Tables 2 and S6). Manual curation of the 121 genes was required to correct the gene models and improve the phylogenetic analysis. Overall, 25 coding sequences (CDSs) were corrected through manual curation. After this step, another 25 genes were renamed multicopper oxidase-like genes (MCO-Like) due to their unusual length (less than 400 residues, while all the Arabidopsis genes ranged from 526 to $588 \mathrm{aa}$ ) and very low RNA-seq support. This analysis produced a final list of 95 multicopper oxidases, which were used for the phylogenetic analysis with the Arabidopsis genes (Table S5 and Figure 5).

Table 2. Gene model curation of putative V. vinifera $L A C$ genes.

\begin{tabular}{ccccccc}
\hline Source & Initial Genes & $\begin{array}{c}\text { Corrected } \\
\text { CDS }\end{array}$ & Split Genes & Merged Genes & $\begin{array}{c}\text { Curated Gene } \\
\text { Models }\end{array}$ & $\begin{array}{c}\text { Identified as } \\
\text { Pseudogenes or } \\
\text { Non-Laccases }\end{array}$ \\
\hline Present in 12X.v1 & 77 & 16 & 2 & 3 & 76 \\
\hline $\begin{array}{c}\text { HMMER search } \\
\text { in 12X.v2 }\end{array}$ & 44 & 4 & 0 & 0 & 44 \\
\hline Total & 121 & 20 & 2 & 3 & 14 \\
\hline
\end{tabular}

The phylogenetic tree in Figure 5 clearly showed that 19 Vitis genes corresponded to multicopper oxidases such as low phosphate root (LPR) genes, ascorbate oxidases (AO), and SKU5 similar (SKS) proteins, while 76 genes were actual LAC isoforms. They could be divided into nine subgroups, 7 of which contained both Vitis and Arabidopsis genes and reproduced the groups already described for Arabidopsis [54,55], while groups 8 and 9 only contained Vitis LACs. Interestingly, the remarkable gene expansion of the grapevine LAC family was primarily due to the two additional Vitis-specific subgroups located on chromosome 18 (Table 3). Due to the lack of a clear correspondence between Vitis and Arabidopsis genes and these additional large groups, LAC isoforms were named according to gene position within the phylogenetic tree, as recommended in [56]. By doing so, the consecutive numbering of LACs from each subgroup was granted.

Table 3. Comparison between Vitis and Arabidopsis groups and subgroups cardinality.

\begin{tabular}{|c|c|c|c|c|c|c|}
\hline Species & LAC (Group 1 to 7) & LAC (Group 8 and 9) & Total LACs & Low Phosphate Root & Ascorbate Oxidases & SKU5 Similar \\
\hline Vitis vinifera & 24 & 52 & 76 & 2 & 5 & 12 \\
\hline Arabidopsis thaliana & 17 & 0 & 17 & 2 & 3 & 19 \\
\hline
\end{tabular}

Even more interesting finding was that all the LACs identified as part of the stilbenoid pathway belonged to group 8 , suggesting a gain of functional specificity of this group related to resveratrol oligomerization. Conversely, LACs related to the lignin network belonged to subgroups 1-3 and one to group 8 . Within the LAC family, the functional subdivision almost entirely corresponded to a phylogenetic difference.

The same pipeline of analysis was applied to characterize the DIR family. The HMMER search on the 12X.v2 genome assembly identified another nine genes in addition to the 32 already annotated as DIR in the 12X.v1 (Table 4). Manual curation of the gene models improved the CDS prediction of eight isoforms. 


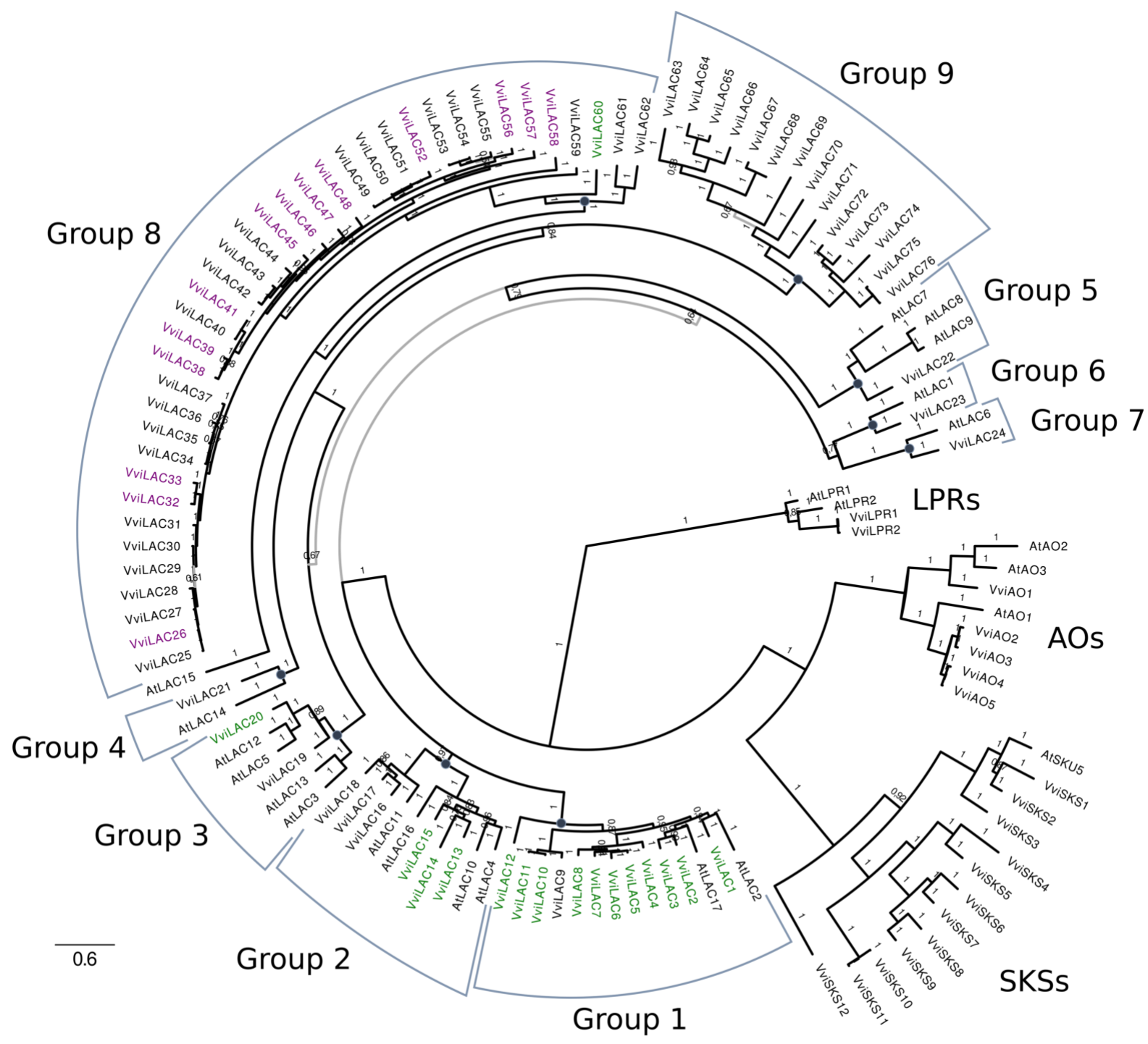

Figure 5. Phylogenetic analysis of the grapevine LAC family and integration with OneGenE results. Bayesian phylogenetic analysis using MrBayes after 3 million generations and average standard deviation of split frequencies of 0.010. LAC genes in the lignin and stilbene OneGeneE networks have green and purple labels, respectively. LAC groups 1-7 have been previously identified in Arabidopsis (all contain Vitis and Arabidopsis sequences). LAC groups 8 and 9 have been newly identified and contain Vitis sequences exclusively. This analysis correctly reassigned some putative LACs to the ascorbate oxidases (AO) and SKU5 similar (SKS) families, while two Vitis genes homologous to the two low phosphate root (LPR) genes in Arabidopsis were used as an outgroup.

Table 4. Gene model curation of putative V. vinifera DIR genes.

\begin{tabular}{cccccc}
\hline Source & Initial Genes & Corrected CDS & Split Genes & Merged Genes & Curated Gene Models \\
\hline Present in 12X.v1 & 32 & 6 & 0 & 0 & 32 \\
\hline HMMER search in 12X.v2 & 9 & 2 & 0 & 0 & 9 \\
\hline Total & 41 & 8 & 0 & 0 & 41 \\
\hline
\end{tabular}

The phylogenetic analysis performed on the 41 and 25 DIR isoforms from Vitis and Arabidopsis, respectively, showed that all putative DIR proteins from Vitis were closely related to an Arabidopsis homolog and could be considered true DIR proteins (Figure 6). 
The previously described DIR groups in Arabidopsis thaliana appear to be recapitulated in general $[57,58]$, however, further subdivisions were suggested by the phylogenetic branches, and a numeric suffix was appended to the groups a, bd, and e accordingly. The bd group was clearly expanded in Vitis with respect to Arabidopsis. The final DIR nomenclature was defined according to gene position within the phylogenetic tree as done for LAC. The integration of the OneGenE functional results and the phylogenetic analysis produced less distinguished group associations, as the DIR isoform related to the stilbenoid network belonged to subgroup bd2 and the orphan VviDIR41. In contrast, those related to the lignin network belonged to subgroup a, bd1, and bd2. VviDIR 12, 13, and 16 and were connected to almost all VviSTSs, while VviDIR41 appeared to belong to a smaller group formed by VviSTS45, 7, and the four clusters, which account for 21 isoforms.

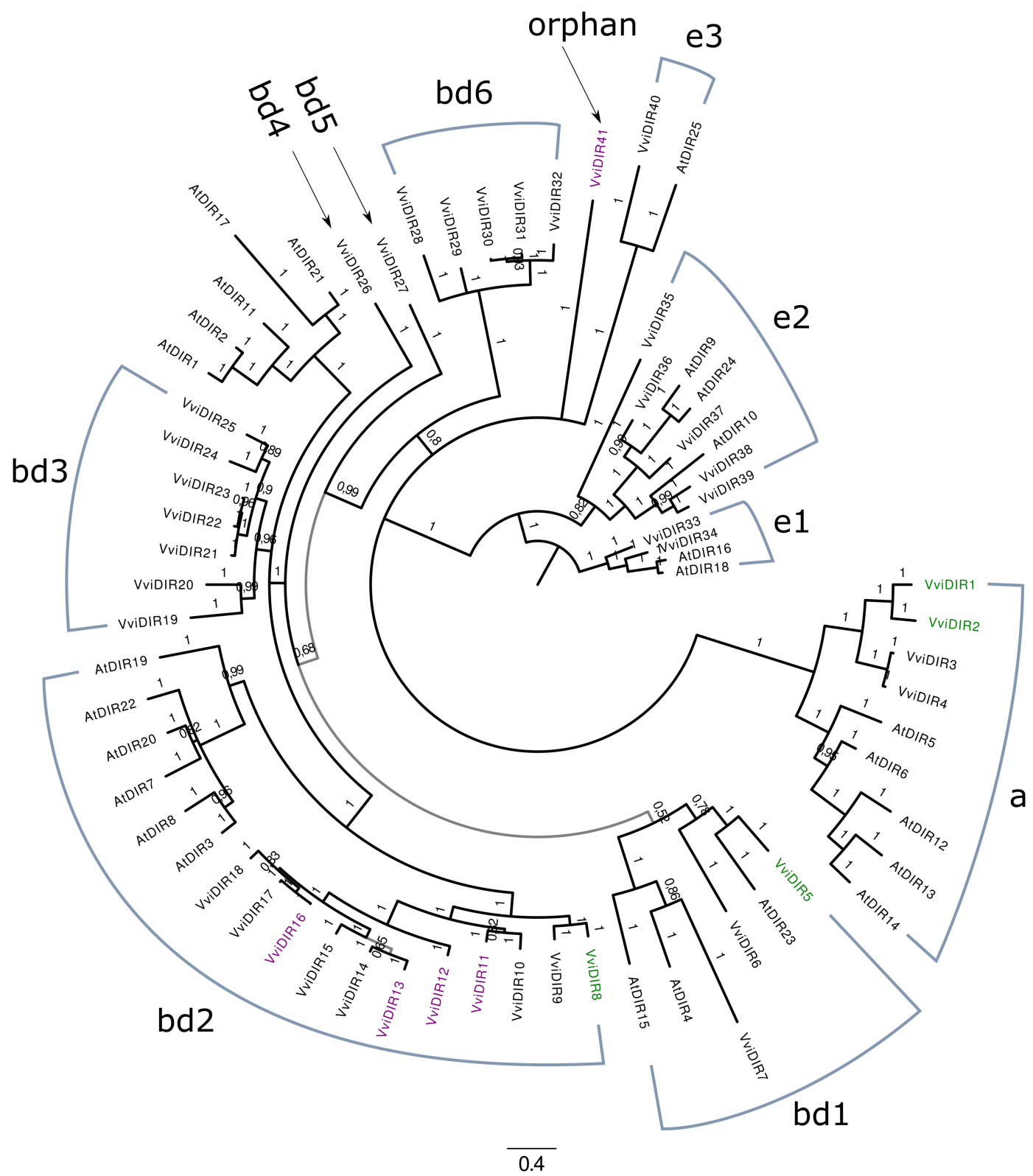

Figure 6. Phylogenetic analysis of the grapevine DIR family and integration with OneGenE results. Bayesian phylogenetic analysis using MrBayes after 1 million generations and average standard deviation of split frequencies of 0.0075. Subgroup e1 was used to root the tree. DIR genes in the lignin and stilbene OneGeneE networks have green and purple labels, respectively. 


\section{Discussion}

The power of applying the PC-based causal inference approach to grapevine transcriptomic data has already been appreciated when expanding local gene networks with $\mathrm{NES}^{2} \mathrm{RA}$ [16]. The strategy to start from a completely connected network and remove edges in two steps, the first based on Pearson's correlation and the second based on conditional independence, allows not only to simplify a co-expression network but also to improve it by removing indirectly linked genes thus discriminating between co-occurrence and functional dependency. The main drawback of NES ${ }^{2}$ RA for the biologist users was the impossibility to perform their expansion analyses themselves due to the time and computational effort requirement for each analysis and despite the availability of the BOINC-based distributed computation platform of TN-GRID [17]. Another drawback was the non-reusability of such results, being GRN specific. OneGenE overcomes these problems by expanding a local gene network made of one gene, computing it for all the genes of the genome, and making them readily available to the user, free to expand the preferred gene network by using the OneGenE lists (Figure 1). The OneGenE approach better accomplishes GO FAIR requirements [3], as the expansion precomputed lists are reusable and have been made Findable and Accessible through the website presented here. An example of Interoperability of the data is provided by the tools that we developed to reconstruct gene networks and have been implemented on the website analysis page to facilitate the usage of OneGenE lists. The architecture of the project is suitable for future improvement and implementation, such as on one side, the re-calculation of all the expansion lists on the upcoming version of the VESPUCCI database when available (Moretto et al., submitted paper), and on the other side, the development of new tools, such as those for network reconstruction and functional enrichment analyses. An improvement of the gene annotation, such as the one promoted through the Vitis Gene Catalogue within the COST Action INTEGRAPE (integrape.eu), is certainly desirable to make the expansion lists more informative for the biologists.

As a proof of concept of the OneGenE approach, we reconstructed the stilbene synthase gene regulatory network (GRN) and compared it with the available knowledge, represented by both experimental evidence [25,49-53] and in silico analyses [13,49]. The results obtained with OneGenE showed an extended overlap with these previous findings (Table 1), confirming its predictive value. In particular, $50 \%$ of the TFs identified by OneGenE as directly connected to VviSTS were supported by previous works. The network visualization highlighted a partitioning of the VviSTSs around three main hubs, namely VviMYB14, the VviWRKY43-53 pair, and the VviMYB13-15-VviWRKY24 triad (Figure 2A), which may reflect different regulatory cascades. Additional genes have been identified as related to STS, in particular, two C3HC4 type Zinc finger factors (36 and 163), which showed to be highly connected within the VviWRKY43-53 subnetwork, and four JAZ (Jasmonate ZIM domain) TFs located in the VviMYB14 subnetwork. A closer look at the list of genes related to STSs (Table S2) revealed that OneGeneE identified many genes involved in JA syntheses, such as allene oxide synthase and 12-oxophytodienoate reductase, and JA signaling, such as two MYC2 TFs and several PR10 genes. The involvement of JA in grapevine response to Plasmopara viticola, especially in resistant genotypes such as Regent, which accumulates stilbenoids as a defense strategy [50], has already been pointed out [59], and the accumulation of resveratrol upon treatment of $V$. vinifera cell cultures with JA has been previously reported [60]. Thus, our analysis may contribute to elucidating the signaling cascade connecting JA and stilbenoid synthesis during pathogen infection by proposing novel candidate genes to be investigated. Moreover, some genes related to salicylic acid signaling have also been identified, as expected, by the known cross-talk of JA and SA in the plant defense to this biotrophic pathogen [59].

Once validated, the list of VviSTS interacting genes have been inspected to identify genes putatively involved in stilbenoid formation, a class of phytoalexins involved in grapevine defense from pathogens and response to abiotic stress such as UV stress [19]. The compounds with the highest fungitoxic activity include $\varepsilon$-viniferins and trans-pterostilbene, which derive from the oxidative dimerization and methylation of resveratrol, respec- 
tively [61]. OneGeneE identified a resveratrol-O-methyltransferase correlated to VviSTS20, also negatively correlated to a glycosyltransferase catalyzing the synthesis of piceid, recognized as a storage form of resveratrol without antifungal properties [20]. The enzymes involved in the oxidative oligomerization of resveratrol are not known at the moment. Thus, we attempted to gain insight through the analogy with another class of polyphenolsthe monolignols - which have been better characterized in Arabidopsis. We noticed that some enzymes contributing to the lignin biosynthetic pathway were also present in the stilbenoid network. These were the LAC and PRX III oxidoreductase enzymatic families involved in monolignol oxidative polymerization [28,31] and the DIR proteins involved in stereospecific positioning of the reactive intermediates [30]. There is evidence that resveratrol units form phenoxyl-radicals upon the action of oxidases such as peroxidase or laccase enzymes, which then condense to form highly polymerized stilbenoids [21,27,62]. Indeed, six VviPrxIII and two LAC genes were recently identified within QTL regions associated with stilbenoid accumulation [26].

Furthermore, fungal laccases could also drive resveratrol oligomerization or degradation during plant-pathogen interactions $[63,64]$, increasing the complexity of the process. Finally, the involvement of dirigent proteins (DIR), similar to those described for the synthesis of optically active lignans and lignins, has been speculated due to the stereospecificity of stilbene oligomers, but not proven thus far [21,27]. We, therefore, reconstructed the lignin network using the OneGenE expansion lists of six MYB TFs that were proposed as involved in lignin biosynthesis in previous work [33]. Interestingly, we obtained two neatly distinguished lists of isoforms for the three enzymatic families putatively involved in stilbenoid and lignin synthesis (Figures 3 and 4): 16 VviLAC, 2 VviPrx III, and 4 VviDIR genes exclusively associated with the lignin network, and 14 VviLAC, 9 VviPrx and 5 VviDIR genes exclusively associated to the stilbenoid network. It is worth pointing out that VviPrxIII07c, identified in the lignin network, is the homolog of AtPrx64-based on the phylogenetic reconstruction of the VviPrxIII gene family [26] — characterized as involved in sclerenchyma lignification [31]. Conversely, VviPrxIII23b and VviPrxIII23c identified in the stilbenoid network belong to the class 23, as does VviPrxIII23a, a dimeric stilbenoid previously found within a QTL region associated with $\omega$-viniferin [26]. The other seven VviPrx genes connected to VviSTS and belonging to classes 01 and 22 represent novel candidates for stilbenoid formation to be further functionally characterized.

For the LAC and DIR families, a genome-wide characterization was carried out using the newly released assembly (12X.v2) and annotation (VCost.v3) of the grapevine genome [42], which allowed to retrieve additional genes and refine them based on RNAseq data (Tables 2 and 4). Phylogenetic analysis performed on the manually curated sequences revealed interesting features of these families (Figures 5 and 6), especially for the $L A C$ one. They are composed of 76 members, 52 of which are localized on chromosome 18. They are clustered into two groups, named eight and nine, which do not have any Arabidopsis homologs (Figure 5, Table 3). We also analyzed the $L A C$ family of Citrus sinensis, formed by 24 isoforms clustering into seven groups as Arabidopsis (not shown), replicating the results from a previously published phylogenetic study [55]. Integrating this analysis with OneGenE results, it appeared that all the stilbenoid-related $L A C$ isoforms belonged to group 8 , while those identified in the lignin network belonged to groups 1 , 2, and 3, and VviLAC60 to group 8 (Figure 5). Five of the group 8 LAC genes, namely VviLAC38-41 and VviLAC46, are located within the Rpv3.3 locus (as defined by the VVIN16 and UDV737 markers [65]) and were all directly connected to VviSTSs. This result supports the observed relationship between this major locus for $P$. viticola resistance present in European resistant grapevines [65] and the accumulation of stilbenoids upon downy mildew infection $[26,50,66]$. The functional divergence of group 8 and $9 L A C$ genes was coherent with the observation that Arabidospsis and Citrus, which did not synthesize stilbenes, did not undergo this family expansion in their genome. Several pseudogenes named VviMCO-Like after our analysis are located in the same region of chromosome 18 , suggesting several events of tandem duplication. The OneGenE analysis did not find 
VviLACs of group 9 as associated with VviSTS. However, it is interesting to report that group 9 VviLAC64 and VviLAC66 are located within the QTL region (centered on the VMC8B5 marker) associated with $\varepsilon$-viniferin, a dimeric stilbenoid, and therefore putatively involved in its oligomerization (Table S5 [26]. DIR isoforms clustered with Arabidopsis ones, despite a limited expansion of the bd2 group (Figure 6). Phylogenetic groups reflected close localization on different chromosomes, suggesting that expansion was gained by gene tandem duplication. Only one isoform appeared distinct from all the others and is located alone on chromosome 4, VviDIR41. This orphan gene is associated with stilbenoids, as bd2 isoforms, while those associated with lignin synthesis are in groups a, and bd1, and VviDIR8 in group bd2.

\section{Conclusions}

We have presented the application of a causality-based approach to reconstruct association gene networks in the grapevine. OneGenE precomputed a ranked list of directly related genes for each gene of the $V$. vinifera genome, which are accessible for download from a dedicated website. Two tools have also been made available for reconstructing gene networks based on a selection of genes of interest for the user. The stilbenoid network, reconstructed from VviSTS genes, has been thoroughly investigated both in its regulation, finding known and novel TFs, and metabolic pathway, identifying genes potentially involved in resveratrol modification and oligomerization. The LAC and DIR family have been characterized on a genome-wide scale and revealed a functional specialization in polyphenols oxidative oligomerization belonging to different pathways, such as those of stilbenoids and lignin biosynthesis. OneGenE proved to be an efficient method able to cope with the complexity of transcriptomic data to identify direct connections among genes and to propose novel candidates for experimental investigations.

Supplementary Materials: The following are available online at https:/ /www.mdpi.com/article/10 .3390 /biom11121744/s1, Figure S1: The Stilbene synthases (STS) network, Table S1: The STS gene family, Table S2: The complete aggregated list of the STS network expansion, Table S3: GO enrichment analysis of the aggregated list of STS network expansion, Table S4: The complete aggregated list of the Lignin gene network, Table S5: GO enrichment analysis of the aggregated list of Lignin gene network, Table S6: The grapevine and Arabidopsis LAC families, Table S7: The grapevine and Arabidopsis DIR families.

Author Contributions: Conceptualization, S.P., G.M., C.M., E.B. and V.C.; Method, G.T., L.R., E.B. and V.C.; Analysis and the OneGenE website G.T., L.R., S.P., E.B. and V.C; Data curation and phylogenetic analysis, D.N.-P. and J.T.M.; Validation, J.T.M., G.M. and S.P.; Network visualization, S.P., C.M. and G.M.; Writing—original draft, S.P., G.M. and C.M., All the authors, contributed to the final writing and reviewing. All authors have read and agreed to the published version of the manuscript.

Funding: The Autonomous Province of Trento (PAT) partially funded this research work under the Accordo di Programma 2018-20. JTM is supported by Grant PGC2018-099449-A-I00 and by the Ramón y Cajal program grant RYC-2017-23645, from the Ministerio de Ciencia, Innovación y Universidades (MCIU, Spain), Agencia Estatal de Investigación (AEI, Spain), and Fondo Europeo de Desarrollo Regional (FEDER, European Union).

Acknowledgments: This article is based on COST Action CA17111 INTEGRAPE, supported by COST (European Cooperation in Science and Technology). Data has been treated and uploaded in public repositories according to the FAIR principles, following the guidelines found at INTEGRAPE. The authors wish to thank all the BOINC volunteers for the computational effort and Tommaso Oss and Nikolas Plattner for building the initial version of the website during their scholar internship.

Conflicts of Interest: The authors declare no conflict of interest. The funders had no role in the study's design, in the analyses or interpretation of data, in the writing of the manuscript, or in the decision to publish the results. 


\section{References}

1. Moretto, M.; Sonego, P.; Pilati, S.; Malacarne, G.; Costantini, L.; Grzeskowiak, L.; Bagagli, G.; Grando, M.S.; Moser, C.; Engelen, K. VESPUCCI: Exploring Patterns of Gene Expression in Grapevine. Front. Plant Sci. 2016, 7, 633. [CrossRef]

2. Wang, Y.; Zhang, R.; Liang, Z.; Li, S. Grape-rna: A database for the collection, evaluation, treatment, and data sharing of grape RNA-seq datasets. Genes 2020, 11, 315. [CrossRef] [PubMed]

3. Wilkinson, M.D.; Dumontier, M.; Aalbersberg, I.J.; Appleton, G.; Axton, M.; Baak, A.; Blomberg, N.; Boiten, J.W.; da Silva Santos, L.B.; Bourne, P.E.; et al. Comment: The FAIR Guiding Principles for scientific data management and stewardship. Sci. Data 2016, 3, 1-9. [CrossRef] [PubMed]

4. Hasty, J.; McMillen, D.; Isaacs, F.; Collins, J.J. Computational studies of gene regulatory networks: In numero molecular biology. Nat. Rev. Genet. 2001, 2, 268-279. [CrossRef] [PubMed]

5. Li, Y.; Pearl, S.A.; Jackson, S.A. Gene Networks in Plant Biology: Approaches in Reconstruction and Analysis. Trends Plant Sci. 2015, 20, 664-675. [CrossRef]

6. Savoi, S.; Wong, D.C.J.; Degu, A.; Herrera, J.C.; Bucchetti, B.; Peterlunger, E.; Fait, A.; Mattivi, F.; Castellarin, S.D. Multi-Omics and Integrated Network Analyses Reveal New Insights into the Systems Relationships between Metabolites, Structural Genes, and Transcriptional Regulators in Developing Grape Berries (Vitis vinifera L.) Exposed to Water Deficit. Front. Plant Sci. 2017, 8, 1124. [CrossRef] [PubMed]

7. Bertazzon, N.; Bagnaresi, P.; Forte, V.; Mazzucotelli, E.; Filippin, L.; Guerra, D.; Zechini, A.; Cattivelli, L.; Angelini, E. Grapevine comparative early transcriptomic profiling suggests that Flavescence dorée phytoplasma represses plant responses induced by vector feeding in susceptible varieties. BMC Genomics 2019, 20, 1-27. [CrossRef]

8. Fajardo, T.V.M.; Quecini, V. Comparative transcriptome analyses between cultivated and wild grapes reveal conservation of expressed genes but extensive rewiring of co-expression networks. Plant Mol. Biol. 2021, 1, 3. [CrossRef]

9. Toups, H.S.; Cochetel, N.; Gray, D.; Cramer, G.R. VviERF6Ls: An expanded clade in Vitis responds transcriptionally to abiotic and biotic stresses and berry development. BMC Genomics 2020, 21, 1-27. [CrossRef]

10. Ghan, R.; Petereit, J.; Tillett, R.L.; Schlauch, K.A.; Toubiana, D.; Fait, A.; Cramer, G.R. The common transcriptional subnetworks of the grape berry skin in the late stages of ripening. BMC Plant Biol. 2017, 17, 1-21. [CrossRef]

11. Hopper, D.W.; Ghan, R.; Schlauch, K.A.; Cramer, G.R. Transcriptomic network analyses of leaf dehydration responses identify highly connected ABA and ethylene signaling hubs in three grapevine species differing in drought tolerance. BMC Plant Biol. 2016, 16, 1-20. [CrossRef] [PubMed]

12. Cramer, G.R.; Cochetel, N.; Ghan, R.; Destrac-Irvine, A.; Delrot, S. A sense of place: Transcriptomics identifies environmental signatures in Cabernet Sauvignon berry skins in the late stages of ripening. BMC Plant Biol. 2020, 20, 1-26. [CrossRef] [PubMed]

13. Wong, D.C.J.; Matus, J.T. Constructing Integrated Networks for Identifying New Secondary Metabolic Pathway Regulators in Grapevine: Recent Applications and Future Opportunities. Front. Plant Sci. 2017, 8, 505. [CrossRef]

14. Wong, D.C.J. Network aggregation improves gene function prediction of grapevine gene co-expression networks. Plant Mol. Biol. 2020. [CrossRef]

15. Spirtes, P.; Glymour, C. An Algorithm for Fast Recovery of Sparse Causal Graphs. Soc. Sci. Comput. Rev. 1991, 9, 62-72. [CrossRef]

16. Malacarne, G.; Pilati, S.; Valentini, S.; Asnicar, F.; Moretto, M.; Sonego, P.; Masera, L.; Cavecchia, V.; Blanzieri, E.; Moser, C. Discovering Causal Relationships in Grapevine Expression Data to Expand Gene Networks. A Case Study: Four Networks Related to Climate Change. Front. Plant Sci. 2018, 9, 1385. [CrossRef] [PubMed]

17. Asnicar, F.; Sella, N.; Masera, L.; Morettin, P.; Tolio, T.; Semeniuta, S.; Moser, C.; Blanzieri, E.; Cavecchia, V. TN-Grid and gene@home project: Volunteer Computing for Bioinformatics. In Proceedings of the International Conference BOINC:FAST 2015, Petrozavodsk, Russia, 14-18 September 2015; Ivashko, E., Ed.; Aachen University: Aachen, Germany, 2015.

18. Blanzieri, E.; Tebaldi, T.; Cavecchia, V.; Asnicar, F.; Masera, L.; Tome, G.; Nigro, E.; Colasurdo, E.; Ciciani, M.; Mazzoni, C.; et al. A Computing System for Discovering Causal Relationships among Human Genes to Improve Drug Repositioning. IEEE Trans. Emerg. Top. Comput. 2020. [CrossRef]

19. Jeandet, P.; Douillet-Breuil, A.C.; Bessis, R.; Debord, S.; Sbaghi, M.; Adrian, M. Phytoalexins from the vitaceae: Biosynthesis, phytoalexin gene expression in transgenic plants, antifungal activity, and metabolism. J. Agric. Food Chem. 2002, 50, 2731-2741. [CrossRef]

20. Pezet, R.; Gindro, K.; Viret, O.; Spring, J.L. Glycosylation and oxidative dimerization of resveratrol are respectively associated to sensitivity and resistance of grapevine cultivars to downy mildew. Physiol. Mol. Plant Pathol. 2004, 65, 297-303. [CrossRef]

21. Mattivi, F.; Vrhovsek, U.; Malacarne, G.; Masuero, D.; Zulini, L.; Stefanini, M.; Mose, C.; Velasco, R.; Guella, G. Profiling of resveratrol oligomers, important stress metabolites, accumulating in the leaves of hybrid Vitis vinifera (Merzling $\times$ Teroldego) genotypes infected with Plasmopara viticola. J. Agric. Food Chem. 2011, 59, 5364-5375. [CrossRef]

22. Vannozzi, A.; Dry, I.B.; Fasoli, M.; Zenoni, S.; Lucchin, M. Genome-wide analysis of the grapevine stilbene synthase multigenic family: Genomic organization and expression profiles upon biotic and abiotic stresses. BMC Plant Biol. 2012, 12, 130. [CrossRef] [PubMed]

23. Gatto, P.; Vrhovsek, U.; Muth, J.; Segala, C.; Romualdi, C.; Fontana, P.; Pruefer, D.; Stefanini, M.; Moser, C.; Mattivi, F.; et al. Ripening and Genotype Control Stilbene Accumulation in Healthy Grapes. J. Agric. Food Chem. 2008, 56, 11773-11785. [CrossRef] [PubMed] 
24. Malacarne, G.; Vrhovsek, U.; Zulini, L.; Masuero, D.; Cestaro, A.; Stefanini, M.; Delledonne, M.; Velasco, R.; Guella, G.; Mattivi, F.; et al. Resistance to Plasmopara viticola is associated with a complex pattern of stilbenoids and with specific host transcriptional responses. BMC Plant Biol. 2011, 11, 1-13. [CrossRef] [PubMed]

25. Höll, J.; Vannozzi, A.; Czemmel, S.; D’Onofrio, C.; Walker, A.R.; Rausch, T.; Lucchin, M.; Boss, P.K.; Dry, I.B.; Bogs, J. The R2R3-MYB transcription factors MYB14 and MYB15 regulate stilbene biosynthesis in Vitis vinifera. Plant Cell 2013, 25, 4135-4149. [CrossRef]

26. Vezzulli, S.; Malacarne, G.; Masuero, D.; Vecchione, A.; Dolzani, C.; Goremykin, V.; Mehari, Z.H.; Banchi, E.; Velasco, R.; Stefanini, M.; et al. The Rpv3-3 haplotype and stilbenoid induction mediate downy mildew resistance in a grapevine interspecific population. Front. Plant Sci. 2019, 10, 1-23. [CrossRef] [PubMed]

27. Keylor, M.H.; Matsuura, B.S.; Stephenson, C.R.J. Chemistry and Biology of Resveratrol-Derived Natural Products. Chem. Rev. 2015, 115, 8976-9027. [CrossRef] [PubMed]

28. Herrero, J.; Esteban-Carrasco, A.; Zapata, J.M. Looking for Arabidopsis thaliana peroxidases involved in lignin biosynthesis. Plant Physiol. Biochem. 2013, 67, 77-86. [CrossRef]

29. Davin, L.B.; Lewis, N.G. Dirigent proteins and dirigent sites explain the mystery of specificity of radical precursor coupling in lignan and lignin biosynthesis. Plant Physiol. 2000, 123, 453-461. [CrossRef]

30. Paniagua, C.; Bilkova, A.; Jackson, P.; Dabravolski, S.; Riber, W.; Didi, V.; Houser, J.; Gigli-Bisceglia, N.; Wimmerova, M.; Budínská, E.; et al. Dirigent proteins in plants: Modulating cell wall metabolism during abiotic and biotic stress exposure. J. Exp. Bot. 2017, 68, 3287-3301. [CrossRef] [PubMed]

31. Tokunaga, N.; Kaneta, T.; Sato, S.; Sato, Y. Analysis of expression profiles of three peroxidase genes associated with lignification in Arabidopsis thaliana. Physiol. Plant. 2009, 136, 237-249. [CrossRef] [PubMed]

32. Ohtani, M.; Demura, T. The quest for transcriptional hubs of lignin biosynthesis: Beyond the NAC-MYB-gene regulatory network model. Curr. Opin. Biotechnol. 2019, 56, 82-87. [CrossRef] [PubMed]

33. Wong, D.C.J.; Schlechter, R.; Vannozzi, A.; Höll, J.; Hmmam, I.; Bogs, J.; Tornielli, G.B.; Castellarin, S.D.; Matus, J.T. A systemsoriented analysis of the grapevine R2R3-MYB transcription factor family uncovers new insights into the regulation of stilbene accumulation. DNA Res. 2016, 23, 451. [CrossRef] [PubMed]

34. Ohman, D.; Demedts, B.; Kumar, M.; Gerber, L.; Gorzsas, A.; Goeminne, G.; Hedenström, M.; Ellis, B.; Boerjan, W.; Sundberg, B. MYB103 is required for FERULATE-5-HYDROXYLASE expression and syringyl lignin biosynthesis in Arabidopsis stems. Plant J. 2013, 73, 63-76. [CrossRef] [PubMed]

35. Yang, C.; Song, J.; Ferguson, A.C.; Klisch, D.; Simpson, K.; Mo, R.; Taylor, B.; Mitsuda, N.; Wilson, Z.A. Transcription factor MYB26 is key to spatial specificity in anther secondary thickening formation. Plant Physiol. 2017, 175, 333-350. [CrossRef] [PubMed]

36. Geng, P.; Zhang, S.; Liu, J.; Zhao, C.; Wu, J.; Cao, Y.; Fu, C.; Han, X.; He, H.; Zhao, Q. MYB20, MYB42, MYB43, and MYB85 regulate phenylalanine and lignin biosynthesis during secondary cell wall formation1[OPEN]. Plant Physiol. 2020, 182, 1272-1283. [CrossRef] [PubMed]

37. Zhong, R.; Richardson, E.A.; Ye, Z.H. The MYB46 transcription factor is a direct target of SND1 and regulates secondary wall biosynthesis in Arabidopsis. Plant Cell 2007, 19, 2776-2792. [CrossRef] [PubMed]

38. Asnicar, F.; Masera, L.; Pistore, D.; Valentini, S.; Cavecchia, V.; Blanzieri, E. OneGenE: Regulatory Gene Network Expansion via Distributed Volunteer Computing on BOINC. In Proceedings of the 27th Euromicro International Conference on Parallel, Distributed and Network-Based Processing (PDP), Pavia, Italy, 13-15 February 2019.

39. Kalisch, M.; Bühlmann, P. Estimating high-dimensional directed acyclic graphs with the PC-algorithm. J. Mach. Learn. Res. 2007, 8, 613-636.

40. Anderson, D.P. BOINC: A System for Public-Resource Computing and Storage. In Proceedings of the 5th IEEE/ ACM International Workshop on Grid Computing, Washington, DC, USA, 8 November 2004; IEEE Computer Society: Washington, DC, USA, 2004; pp. 4-10.

41. Grimplet, J.; Van Hemert, J.; Carbonell-Bejerano, P.; Díaz-Riquelme, J.; Dickerson, J.; Fennell, A.; Pezzotti, M.; Martínez-Zapater, J.M. Comparative analysis of grapevine whole-genome gene predictions, functional annotation, categorization and integration of the predicted gene sequences. BMC Res. Notes 2012, 5, 213. [CrossRef]

42. Canaguier, A.; Grimplet, J.; di Gaspero, G.; Scalabrin, S.; Duchêne, E.; Choisne, N.; Mohellibi, N.; Guichard, C.; Rombauts, S.; le Clainche, I.; et al. A new version of the grapevine reference genome assembly (12X.v2) and of its annotation (VCost.v3). Genomics Data 2017, 14, 56-62. [CrossRef]

43. Shannon, P.; Markiel, A.; Ozier, O.; Baliga, N.S.; Wang, J.T.; Ramage, D.; Amin, N.; Schwikowski, B.; Ideker, T. Cytoscape: A software environment for integrated models of biomolecular interaction networks. Genome Res. 2003, 13, 2498-2504. [CrossRef] [PubMed]

44. Franz, M.; Lopes, C.T.; Huck, G.; Dong, Y.; Sumer, O.; Bader, G.D. Cytoscape.js: A graph theory library for visualisation and analysis. Bioinformatics 2016, 32, 309-311. [CrossRef] [PubMed]

45. Alexa, A.; Rahnenfuehrer, J. Gene Set Enrichment Analysis with topGO. 2015, pp. 1-27. Available online: https://bioconductor. org/packages/release/bioc/vignettes/topGO/inst/doc/topGO.pdf (accessed on 10 March 2020).

46. Bailey, T.L. STREME: Accurate and versatile sequence motif discovery. Bioinformatics 2021, 37, 2834-2840. [CrossRef] [PubMed]

47. Lee, E.; Helt, G.A.; Reese, J.T.; Munoz-Torres, M.C.; Childers, C.P.; Buels, R.M.; Stein, L.; Holmes, I.H.; Elsik, C.G.; Lewis, S.E. Web Apollo: A web-based genomic annotation editing platform. Genome Biol. 2013, 14. [CrossRef] [PubMed] 
48. Huelsenbeck, J.P.; Ronquist, F. MRBAYES: Bayesian inference of phylogenetic trees. Bioinformatics 2001, 17, 754-755. [CrossRef]

49. Vannozzi, A.; Wong, D.C.J.; Höll, J.; Hmmam, I.; Matus, J.T.; Bogs, J.; Ziegler, T.; Dry, I.; Barcaccia, G.; Lucchin, M. Combinatorial Regulation of Stilbene Synthase Genes by WRKY and MYB Transcription Factors in Grapevine (Vitis vinifera L.). Plant Cell Physiol. 2018, 59, 1043-1059. [CrossRef]

50. Eisenmann, B.; Czemmel, S.; Ziegler, T.; Buchholz, G.; Kortekamp, A.; Trapp, O.; Rausch, T.; Dry, I.; Bogs, J. Rpv3-1 mediated resistance to grapevine downy mildew is associated with specific host transcriptional responses and the accumulation of stilbenes. BMC Plant Biol. 2019, 19, 1-17. [CrossRef]

51. Wang, D.; Jiang, C.; Liu, W.; Wang, Y.; Hancock, R. The WRKY53 transcription factor enhances stilbene synthesis and disease resistance by interacting with MYB14 and MYB15 in Chinese wild grape. J. Exp. Bot. 2020, 71, 3211-3226. [CrossRef] [PubMed]

52. Orduña, L.; Li, M.; Navarro-Payá, D.; Zhang, C.; Santiago, A.; Romero, P.; Ramšak, Ž.; Gruden, K.; Höll, J.; Merz, P.; et al. Major orchestration of shikimate, early phenylpropanoid and stilbenoid pathways by Subgroup 2 R2R3-MYBs in grapevine. bioRxiv 2021, 1-16. [CrossRef]

53. Corso, M.; Vannozzi, A.; Maza, E.; Vitulo, N.; Meggio, F.; Pitacco, A.; Telatin, A.; D’Angelo, M.; Feltrin, E.; Negri, A.S.; et al. Comprehensive transcript profiling of two grapevine rootstock genotypes contrasting in drought susceptibility links the phenylpropanoid pathway to enhanced tolerance. J. Exp. Bot. 2015, 66, 5739-5752. [CrossRef]

54. Turlapati, P.V.; Kim, K.W.; Davin, L.B.; Lewis, N.G. The laccase multigene family in Arabidopsis thaliana: Towards addressing the mystery of their gene function(s). Planta 2011, 233, 439-470. [CrossRef]

55. Xu, X.; Zhou, Y.; Wang, B.; Ding, L.; Wang, Y.; Luo, L.; Zhang, Y.; Kong, W. Genome-wide identification and characterization of laccase gene family in Citrus sinensis. Gene 2019, 689, 114-123. [CrossRef]

56. Grimplet, J.; Adam-Blondon, A.F.; Bert, P.F.; Bitz, O.; Cantu, D.; Davies, C.; Delrot, S.; Pezzotti, M.; Rombauts, S.; Cramer, G.R. The grapevine gene nomenclature system. BMC Genomics 2014, 15. [CrossRef] [PubMed]

57. Ralph, S.; Park, J.Y.; Bohlmann, J.; Mansfield, S.D. Dirigent proteins in conifer defense: Gene discovery, phylogeny, and differential wound- and insect-induced expression of a family of DIR and DIR-like genes in spruce (Picea spp.). Plant Mol. Biol. 2006, 60, 21-40. [CrossRef] [PubMed]

58. Ralph, S.G.; Jancsik, S.; Bohlmann, J. Dirigent proteins in conifer defense II: Extended gene discovery, phylogeny, and constitutive and stress-induced gene expression in spruce (Picea spp.). Phytochemistry 2007, 68, 1975-1991. [CrossRef] [PubMed]

59. Guerreiro, A.; Figueiredo, J.; Sousa Silva, M.; Figueiredo, A. Linking jasmonic acid to grapevine resistance against the biotrophic oomycete Plasmopara viticola. Front. Plant Sci. 2016, 7, 565. [CrossRef] [PubMed]

60. Tassoni, A.; Fornalè, S.; Franceschetti, M.; Musiani, F.; Michael, A.J.; Perry, B.; Bagni, N. Jasmonates and Na-orthovanadate promote resveratrol production in Vitis vinifera cv. Barbera cell cultures. New Phytol. 2005, 166, 895-905. [CrossRef] [PubMed]

61. Pezet, R.; Gindro, K.; Viret, O.; Richter, H. Effects of resveratrol, viniferins and pterostilbene on Plasmopara viticola zoospore mobility and disease development. Vitis -J. Grapevine Res. 2004, 43, 145-148.

62. Cichewicz, R.H.; Kouzi, S.A. Resveratrol oligomers: Structure, chemistry, and biological activity. Stud. Nat. Prod. Chem. 2002, 26, 507-579. [CrossRef]

63. Pezet, R.; Pont, V.; Hoang-Van, K. Evidence for oxidative detoxication of pterostilbene and resveratrol by a laccase-like stilbene oxidase produced by Botrytis cinerea. Physiol. Mol. Plant Pathol. 1991, 39, 441-450. [CrossRef]

64. Breuil, A.C.; Jeandet, P.; Adrian, M.; Chopin, F.; Pirio, N.; Meunier, P.; Bessis, R. Characterization of a pterostilbene dehydrodimer produced by laccase of Botrytis cinerea. Phytopathology 1999, 89, 298-302. [CrossRef]

65. Di Gaspero, G.; Copetti, D.; Coleman, C.; Castellarin, S.D.; Eibach, R.; Kozma, P.; Lacombe, T.; Gambetta, G.; Zvyagin, A.; Cindrić, P.; et al. Selective sweep at the Rpv3 locus during grapevine breeding for downy mildew resistance. Theor. Appl. Genet. 2012, 124, 277-286. [CrossRef] [PubMed]

66. Chitarrini, G.; Soini, E.; Riccadonna, S.; Franceschi, P.; Zulini, L.; Masuero, D.; Vecchione, A.; Stefanini, M.; Di Gaspero, G.; Mattivi, F.; et al. Identification of Biomarkers for Defense Response to Plasmopara viticola in a Resistant Grape Variety. Front. Plant Sci. 2017, 8, 1524. [CrossRef] [PubMed] 